\title{
Hearing screening outcomes in pediatric critical care survivors: a 1-year report
}

\author{
Pattita Suwannatrai ${ }^{1}$, Chanapai Chaiyakulsil ${ }^{2}$ \\ ${ }^{1}$ Department of Pediatrics, Faculty of Medicine, Thammasat University, Pathumthani; ${ }^{2}$ Division of Pediatric Critical Care, Department of Pediatrics, Thammasat \\ University Hospital, Faculty of Medicine, Thammasat University, Pathumthani, Thailand
}

Background: Hearing loss is a potentially serious complication that can occur after surviving a critical illness. Study on screening for hearing problems in pediatric critical care survivors beyond the neonatal period is lacking. This study aimed to identify the prevalence of abnormal hearing screening outcomes using transitory evoked otoacoustic emission (TEOAE) screening in children who survived critical illness and to find possible associating factors for abnormal hearing screening results.

Methods: This study was a single-center, prospective, observational study. All children underwent otoscopy to exclude external and middle ear abnormalities before undergoing TEOAE screening. The screening was conducted before hospital discharge. Descriptive statistics, chi-square, and logistic regression tests were used for data analysis.

Results: A total of 92 children were enrolled. Abnormal TEOAE responses were identified in 26 participants (28.3\%). Children with abnormal responses were significantly younger than those with normal responses with a median age of 10.0 months and 43.5 months, respectively $(\mathrm{P}<0.001)$. Positive association with abnormal responses was found in children younger than 12 months of age (adjusted odds ratio [OR], 3.07; 95\% confidence interval $[\mathrm{Cl}], 1.06-8.90)$ and children with underlying genetic conditions (adjusted $\mathrm{OR}, 6.95 ; 95 \% \mathrm{Cl}, 1.49-32.54$ ).

Conclusions: Our study demonstrates a high prevalence of abnormal TEOAE screening responses in children surviving critical illness, especially in patients younger than 12 months of age. More extensive studies should be performed to identify the prevalence and associated risk factors of hearing problems in critically ill children.

Key Words: critical care outcomes; diagnostic screening program; otoacoustic emission; pediatrics; survivors

\section{INTRODUCTION}

Hearing loss can serve as a potentially disastrous clinical problem in children surviving critical illness. Hearing dysfunction is usually classified into either conductive, sensorineural, or mixed type. Conductive hearing loss is related to abnormal sound conduction into the external and middle ear. In contrast, sensorineural loss results from damage of the inner ear or auditory nerve tracts $[1,2]$.

\section{Original Article}

Received: June 28, 2021

Revised: December 22, 2021

Accepted: January 13, 2022

\section{Corresponding author}

Chanapai Chaiyakulsil

Division of Pediatric Critical Care, Department of Pediatrics, Faculty of Medicine, Thammasat University, 95 Phahol Yothin Rd., Klong-Neung, Klong-Luang, Pathumthani 12120, Thailand

Tel: +66-2926-9514

E-mail: chanapai.chai@hotmail.com

Copyright $@ 2022$ The Korean Society of Critical Care Medicine

This is an Open Access article distributed under the terms of Creative Attributions Non-Commercial License (https://creativecommons.org/li-censes/by-nc/4.0/) which permits unrestricted noncommercial use, distribution, and reproduction in any medium, provided the original work is properly cited. 
Pediatric intensive care unit (PICU) survivors are exposed to multiple factors resulting in hearing dysfunction. Factors such as endotracheal tube securing techniques or positive pressure ventilation via masks can result in external ear damage, obstruction, and inadvertent hearing dysfunction [1]. Head trauma diagnosis can lead to tympanic membrane perforation and/or injury of the auditory canal or inner ear labyrinth, resulting in conductive and sensorineural hearing loss [3]. Local ear infection, systemic infection, and neurologic infection can also cause endotoxin-related damage to the inner ear [4-6]. Antimicrobials such as aminoglycosides can damage nonregenerative outer hair cells in the cochlear basal turn, resulting in permanent high-frequency sensorineural hearing damage $[7,8]$. Other medications such as vancomycin, diuretics, cyclooxygenase inhibitors, and chemotherapeutic agents are also associated with hearing loss [1,9-11]. Procedures such as lumbar puncture are also implicated with hearing loss due to endolymphatic hydrops [12].

Routine audiological screening is widely accepted in normal neonates and is universally conducted in critically ill neonates as well. A two-stage protocol starting with transitory evoked otoacoustic emission (TEOAE) is initially used and followed by auditory brainstem responses (ABRs) in individuals with abnormal TEOAE screening responses. Both are validated tools for screening hearing dysfunction in neonates and children [13-15]. Both tools and otoscopy are used to detect hearing loss in critically ill neonates, with the prevalence ranging from $0.2 \%$ to $34.1 \%$ [16-20]. A study in critically ill adults also revealed the prevalence of hearing dysfunction using TEOAE to be as high as $37.3 \%$ [21]. To our knowledge, no study concerning hearing screening outcomes in critically ill children beyond the neonatal period has been conducted. Under-identification and failure to conduct appropriate management of hearing loss in children have an extensive economic impact as well, and also have potential detrimental effects on the individual child's education, speech development, socialization, and emotional health [22].

As a part of a quality improvement service in our hospital, the post-intensive care morbidities monitoring service plans for physical, mental, cognitive, and social impairments after PICU discharge were jointly designed by pediatric intensivists, pediatric intensive care nurses, and developmental-behavioral pediatricians at our hospital. A hearing screening protocol was initiated as a part of this service plan along with this study in an attempt to alleviate the possible impacts of hearing loss. This study aimed to identify the prevalence of abnormal hear-

\section{KEY MESSAGES}

- Approximately $28.3 \%$ of the participants were found to have abnormal transitory evoked otoacoustic emission (TEOAE) screening responses.

- Associations were found in children younger than 12 months of age and in children with genetic comorbidities.

ing screening responses using TEOAE in PICU survivors aged from one month to 15 years of age and to find possible associating factors for abnormal hearing screening.

\section{MATERIALS AND METHODS}

\section{Study Design}

This study was conducted as a prospective, observational study. The Ethics Committee of Human Research approved this research in compliance with the Declaration of Helsinki (No. MTU-EC-PE-6-068/62). Informed consent and assent were obtained from all parents and children participating in this study.

\section{Participants}

All children aged one month to 15 years of age admitted to the PICU for more than 24 hours from October 2019 to October 2020 were enrolled. Our center is a tertiary care university hospital, which also serves as a referral center. Our PICU is a sixbed, mixed medical and surgical intensive care unit caring for infants and children from one month to 15 years of age. The annual admission rate of our PICU is approximately 150 to 200 children. Exclusion criteria for the study included children with previously abnormal TEOAE or ABR hearing screening tests, a previous history of hearing loss, abnormal auricular figures, were unable to perform TEOAE, and/or refused consent.

\section{Data Collection}

Hearing screening was performed as early as possible after PICU discharge and before hospital discharge in a quiet room to reduce ambient noise. All participants underwent otoscopic examination performed by the investigators to rule out possible external ear and middle ear problems such as otitis media, tympanic membrane perforation, and/or cerumen impaction, as these conditions can lead to abnormal TEOAE responses that are unrelated to PICU treatment and environment. Chil- 
dren with abnormal otoscopy examinations were subjected to treatment before conducting TEOAE screening. After treatment for abnormal otoscopic examinations, TEOAE was performed by certified audiologists using the Sentiero TEOAE screener (Screening/Diagnostic; Path Medical Solution, Germering, Germany) to assess hearing function. TEOAEs were measured in both ears of each individual. The non-linear protocol was used at a stimulus level of $85 \mathrm{~dB}$ peak equivalent sound pressure level. Short-term acoustic stimuli without direct component at $0.7-6 \mathrm{kHz}$ were conveyed to the cochlea and the resulting cochlear response was recorded. Normal otoacoustic emission was reported as "pass" and the absence of otoacoustic emission was reported as "refer." Admission diagnosis, demographic data, antimicrobial use, diuretic use, length of hospital stay, and length of PICU stay were collected from the case files.

\section{Statistical Analysis}

Demographic data were analyzed using median and percentages. Categorical data were analyzed using chi-square tests to determine differences between the rates of abnormal hearing screening responses within each variable. Univariate and multivariate logistic regression were used to identify associating factors for an abnormal hearing screening. Crude odds ratio (OR) and adjusted OR were reported along with the 95\% confidence interval (CI). Factors for confounder adjustment were potential factors that led to possible hearing dysfunction obtained from univariate analysis. A P-value $<0.05$ was considered statistically significant. All statistical analyses were performed using SPSS ver. 24 (IBM Corp., Armonk, NY, USA).

\section{RESULTS}

\section{Demographic Data}

A total of 106 participants fulfilled the inclusion criteria during the study period. Three participants were excluded due to a history of abnormal hearing tests, ten patients refused consent for medical information disclosure, and one participant had abnormal auricular figures. Thus, 92 participants were included for analysis (Figure 1). Forty-seven participants (51.1\%) were male. The median age of the cohort was 28.0 months with an interquartile range (IQR) of 8.2-72.2 months. Approximately 29 children (31.5\%) were younger than 12 months of age. Underlying comorbidities were identified in 56 children (60.8\%) with the majority being cardiac comorbidities (34 children; $37.0 \%)$. Twenty-one children (22.8\%) were previously diagnosed with neurological deficits and nine children (9.8\%) had underlying genetic comorbidities.

The most common admission diagnosis was of the cardiology group (38 children, $41.3 \%$ ), mostly being post-operative cardiac surgery and congestive heart failure. Twenty-seven children (29.3\%) were diagnosed with systemic infection and five children (5.4\%) were diagnosed with a neurologic infection. Traumatic brain injury was diagnosed in four children (4.3\%).

Sixty-five participants (70.6\%) were mechanically ventilated. All participants received orotracheal intubation. The median number of ventilator support days was 5.0 days (IQR, 2.0-10.0 days). Twenty-seven participants (29.3\%) were mechanically ventilated for more than seven days. Diuretics were used in 42 children (45.6\%). Approximately $35.7 \%$ of the cohort (33 children) received at least one ototoxic antimicrobial. Within the subgroup of children receiving aminoglycosides (five children, 5.4\%), all received medication for greater than seven days, but none received the medication for greater than 14 days. Lumbar puncture was performed in six individuals (6.5\%). The median PICU stay was 5.0 days (IQR, 3.0-11.0 days), and a total of 42 participants (45.6\%) experienced prolonged PICU stays of greater than 7 days. The median Pediatric Risk of Mortality (PRISM) III score was 2.5 (IQR, 0.0-6.7) [23]. Inotropic support was required in 50 children (54.3\%)

150 Children aged 1 month to 15 years admitted to PICU during the study period

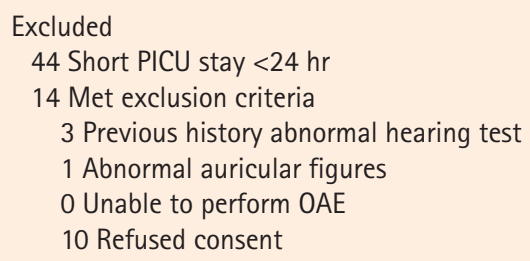

92 Study population
66 Normal OAE screening $(71.7 \%)$
26 Abnormal OAE screening (28.3\%)
Figure 1. Population flowchart. PICU: pediatric intensive care unit; OAE: otoacoustic emission. 
with a median vasoactive inotropic score (VIS) of 10.0 (IQR, 6.7-26.0) [24]. The median hospital stay was 15 days (IQR, 11.0-28.0 days). No family history of hearing problems was found within this cohort. Demographic data of the participants are summarized in Table 1.
Hearing Screening Results and Associating Factors of Abnormal Hearing Screening Responses

None of the participants had abnormal findings on otoscopic examination conducted before TEOAE screening. The median time for TEOAE testing was 7.0 days (IQR 3.0-9.0 days) after PICU discharge. Abnormal TEOAE screening responses were identified in 26 participants (28.3\%). Participants with abnor-

Table 1. Demographic data of the participants

\begin{tabular}{|c|c|c|c|}
\hline Variable & All participants $(n=92)$ & Normal TEOAE screening $(n=66)$ & Refer TEOAE $(n=26)$ \\
\hline Age $(\mathrm{mo})^{a}$ & $28.0(8.2-72.2)$ & $43.5(9.0-83.7)$ & $10.0(7.0-28.7)$ \\
\hline Age $<12 \mathrm{mo}^{\mathrm{a}}$ & $29(31.5)$ & $16(24.2)$ & $13(50.0)$ \\
\hline \multicolumn{4}{|l|}{ Sex } \\
\hline Female & $45(48.9)$ & $32(48.5)$ & $13(50.0)$ \\
\hline Male & $47(51.1)$ & $34(51.5)$ & $13(50.0)$ \\
\hline \multicolumn{4}{|l|}{ Underlying disease } \\
\hline None & $36(39.2)$ & $28(42.4)$ & 8 (30.8) \\
\hline Cardiology & $34(37.0)$ & $26(39.4)$ & $8(30.8)$ \\
\hline Genetic $^{b}$ & $9(9.8)$ & $3(4.5)$ & $6(23.1)$ \\
\hline Pulmonology & $5(5.4)$ & $4(6.1)$ & $1(3.8)$ \\
\hline Gastrointestinal & $4(4.3)$ & $2(3.0)$ & $2(7.7)$ \\
\hline Neurology & $4(4.3)$ & $3(4.5)$ & $1(3.8)$ \\
\hline Nephrology & $3(3.3)$ & $1(1.5)$ & $2(7.7)$ \\
\hline Endocrine & $1(1.1)$ & $1(1.5)$ & - \\
\hline Previous neurologic deficit & $21(22.8)$ & 13 (19.7) & 8 (30.7) \\
\hline Genetic problem $^{a}$ & $9(9.8)$ & $3(4.5)$ & $6(23.1)$ \\
\hline \multicolumn{4}{|l|}{ Admission diagnosis } \\
\hline Cardiology & $38(41.3)$ & $30(45.5)$ & 8 (30.8) \\
\hline Pulmonology & $23(25.0)$ & $14(21.2)$ & $9(34.6)$ \\
\hline Neurology & $13(14.1)$ & $11(16.8)$ & $2(7.7)$ \\
\hline Gastrointestinal & $7(7.6)$ & $3(4.5)$ & $4(15.4)$ \\
\hline Infectious & $4(4.3)$ & $4(6.1)$ & - \\
\hline Nephrology & $2(2.2)$ & - & $2(7.7)$ \\
\hline Endocrinology & $2(2.2)$ & $1(1.5)$ & $1(3.8)$ \\
\hline Genetic & $1(1.1)$ & $1(1.5)$ & - \\
\hline Trauma & $1(1.1)$ & $1(1.5)$ & - \\
\hline Allergy & $1(1.1)$ & $1(1.5)$ & - \\
\hline Systemic infection & 27 (29.3) & $16(24.2)$ & $11(42.3)$ \\
\hline Neurologic infection & $5(5.4)$ & $4(6.1)$ & $1(3.8)$ \\
\hline Traumatic brain injury & $4(4.3)$ & $3(4.5)$ & $1(3.8)$ \\
\hline Medical diagnosis & $30(32.6)$ & $23(34.8)$ & 7 (26.9) \\
\hline \multicolumn{4}{|l|}{ Respiratory support } \\
\hline None & $6(6.5)$ & $6(9.1)$ & - \\
\hline Oxygen cannula & $15(16.3)$ & $9(13.6)$ & $6(23.1)$ \\
\hline Oxygen collar mask & $1(1.1)$ & - & $1(3.8)$ \\
\hline High flow oxygen cannula & $2(2.2)$ & $1(1.5)$ & $1(3.8)$ \\
\hline Non-invasive mechanical ventilator & $3(3.3)$ & $3(4.5)$ & - \\
\hline Mechanical ventilator & $65(70.6)$ & 47 (71.3) & $18(69.2)$ \\
\hline
\end{tabular}

(Continued to the next page) 
Table 1. Continued

\begin{tabular}{lccc}
\hline Variable & All participants $(n=92)$ & Normal TEOAE screening $(n=66)$ & Refer TEOAE $(n=26)$ \\
\hline PRISM III score & $2.5(0.0-6.7)$ & $2(0.0-7.2)$ & $3.5(0.7-6.5)$ \\
Inotropic support & $50(54.3)$ & $33(50.0)$ & $17(65.3)$ \\
Median VIS & $10.0(6.7-26.0)$ & $10.5(6.5-34.5)$ & $10.0(6.7-12.5)$ \\
Median ventilator day & $5.0(2.0-10.0)$ & $5.0(2.0-10.0)$ & $6.5(3.0-11.7)$ \\
Ventilator support $>7$ days & $27(29.3)$ & $17(25.7)$ & $10(38.5)$ \\
Median PICU stay (day) & $5.0(3.0-11.0)$ & $5.5(3.0-11.0)$ & $5.0(3.7-10.7)$ \\
PICU stay > 7 days & $42(45.6)$ & $31(47.0)$ & $11(42.3)$ \\
Median hospital stay (day) & $15.0(11.0-28.0)$ & $15.0(10.0-27.2)$ & $19.0(11.7-31.7)$ \\
Hospital stay $>14$ days & $50(54.3)$ & $34(51.5)$ & $16(61.5)$ \\
Ototoxic antibiotics & $33(35.7)$ & $23(34.8)$ & $10(38.4)$ \\
$\quad$ Vancomycin & $25(27.1)$ & $20(30.3)$ & $5(19.2)$ \\
Aminoglycoside & $5(5.4)$ & $2(3.0)$ & $3(11.5)$ \\
Colistin & $3(3.2)$ & $1(1.5)$ & $2(7.7)$ \\
Diuretics use & $42(45.6)$ & $29(43.9)$ & $13(50.0)$ \\
Lumbar puncture & $6(6.5)$ & $4(6.0)$ & $2(7.7)$ \\
\hline
\end{tabular}

Values are presented as median (interquartile range) or number (\%).

TEOAE: transitory evoked otoacoustic emission; PRISM: Pediatric Risk of Mortality [23]; VIS: vasoactive inotropic score; PICU: pediatric intensive care unit.

${ }^{a} \mathrm{P}<0.05$; ${ }^{b}$ Four patients had both genetic underlying conditions and cardiac underlying conditions, thus were counted into both categories leading cumulative data exceeded 100; ${ }^{\mathrm{C} D o p a m i n e}(\mu \mathrm{g} / \mathrm{kg} / \mathrm{min})+$ dobutamine $(\mu \mathrm{g} / \mathrm{kg} / \mathrm{min})+(10 \times$ milrinone $[\mu \mathrm{g} / \mathrm{kg} / \mathrm{min}])+(100 \times$ epinephrine $[\mu \mathrm{g} / \mathrm{kg} / \mathrm{min}])+(100 \times n o r e p i n e p h r i n e ~[\mu g / \mathrm{kg} /$ $\min ])+(10 x t e r l i p r e s s i n ~ d o s e ~[\mu \mathrm{g} / \mathrm{min}])[24]$.

mal TEOAE were significantly younger than those with normal TEOAE responses with a median age of 10.0 months (IQR, 7.0-28.7 months) and 43.5 months (IQR, 9.0-83.7 months), respectively $(\mathrm{P}<0.001)$. Significantly higher proportions of abnormal TEOAE responses were found in children younger than 12 months of age $(50.0 \%$ vs. $24.2 \%$; $=0.017)$ with crude OR of 3.12 (95\% CI, 1.20-8.10; $\mathrm{P}=0.017$ ). Children with genetic comorbidities were more likely to have abnormal TEOAE responses (23.1\% vs. $4.5 \%$; $\mathrm{P}=0.007)$ with a crude $\mathrm{OR}$ of 6.30 (95\% CI, 1.44-27.52; $\mathrm{P}=0.007$ ). Previous neurological deficits, systemic infection, neurologic infection, and traumatic brain injury were not associated with abnormal TEOAE responses. Respiratory support devices, mechanical ventilation use, and inotropic utilization were comparable among groups.

The severity among participants with and without abnormal TEOAE responses measured by PRISM III were not significantly different (3.5 [IQR, 0.7-6.5] vs. 2.0 [IQR, 0.0-7.2]; $\mathrm{P}=0.905$ ). The median VIS, PICU duration, mechanical ventilation duration, and hospital duration were not significantly different among groups. There was no statistically significant difference in ototoxic antimicrobial utilization in patients with and without abnormal TEOAE responses (34.8\% vs. $38.4 \%$, respectively; $\mathrm{P}=0.745$ ). In the subgroup of children receiving aminoglycosides, there was a higher proportion of children having abnormal TEOAE responses when compared to those who had nor- mal responses, but this finding was not statistically significant (11.5\% vs. $3.0 \%$; $\mathrm{P}=0.107$; crude OR, 4.17 ; 95\% CI, 0.65-26.59). Diuretics were used in 29 children (43.9\%) with normal TEO$\mathrm{AE}$ responses and 13 children (50.0\%) with abnormal TEOAE responses $(\mathrm{P}=0.599)$. Demographic data, treatment, and severity among groups are described in Table 1 . Univariate analysis is demonstrated in Table 2.

After adjustment for age, genetic comorbidities, and aminoglycoside utilization, children younger than 12 months of age were significantly associated with abnormal TEOAE responses with an adjusted OR of 3.07 (95\% CI, 1.06-8.90; P=0.039). Children with genetic comorbidities were also significantly associated with abnormal TEOAE responses (adjusted OR, 6.95; 95\% CI, 1.49-32.54; $\mathrm{P}=0.014$ ). Multivariate logistic regression analyses are summarized in Table 3.

\section{DISCUSSION}

The prevalence of abnormal TEOAE screening responses in this cohort was substantially high at $28.3 \%$. To our knowledge, this study is the first to date aimed to identify the prevalence of abnormal TEOAE screening responses in critically ill children beyond the neonatal period. Results shown here are slightly lower than what was found in a study of critically ill adults by Hamill-Ruth et al. [21] that reported abnormal TEOAE re- 
Table 2. Univariate analysis of associating factors for abnormal TEOAE responses

\begin{tabular}{lcc}
\hline Variable & Crude odds ratio (95\% Cl) & P-value \\
\hline Age $<12$ mo & $3.12(1.20-8.10)$ & 0.017 \\
Female & $0.94(0.38-2.33)$ & 0.896 \\
Neurologic abnormality & $1.81(0.65-5.08)$ & 0.255 \\
Genetic abnormality & $6.30(1.44-27.52)$ & 0.007 \\
Systemic infection & $2.29(0.88-5.99)$ & 0.087 \\
Neurologic infection & $0.62(0.07-5.82)$ & 0.673 \\
Traumatic brain injury & $0.84(0.08-8.46)$ & 0.882 \\
Lumbar puncture & $1.29(0.22-7.52)$ & 0.775 \\
PICU stay $>7$ day & $0.83(0.33-2.07)$ & 0.686 \\
Hospital stay $>14$ day & $1.51(0.60-3.80)$ & 0.385 \\
Ventilator support $>7$ day & $1.80(0.69-4.72)$ & 0.228 \\
Inotropic support & $1.89(0.74-4.84)$ & 0.182 \\
Ototoxic antibiotics & $1.17(0.46-2.99)$ & 0.745 \\
Aminoglycoside use & $4.17(0.65-26.59)$ & 0.107 \\
Diuretic use & $1.28(0.51-3.17)$ & 0.599 \\
\hline
\end{tabular}

TEOAE: transitory evoked otoacoustic emission; $\mathrm{Cl}$ : confidence interval; PICU: pediatric intensive care unit.

sponses at $37.3 \%$. Compared to the two large cohort studies in critically ill neonates by van Dommelen et al. $[16,17]$ in 2010 and 2015, this cohort revealed a higher prevalence of abnormal hearing tests. The possible explanation might be due to different testing methods used in the studies and different population groups. Our study used TEOAE, which is more readily available in our center, rather than the $A B R$, which required a more extended period of appointment.

Critical illness, antimicrobials, ototoxic medications, and loud ambient noise in the intensive care environment were reported to negatively impact PICU patient hearing and might even cause hearing impairment [1,3-12,25]. Impaired auditory acuity in PICU survivors is a potentially disastrous condition that can affect multiple aspects of the children's lives post-intensive care. A study by the World Health Organization reported an impact on speech development, socialization, and mental and emotional health of the child with hearing dysfunction [22]. Routine audiological screening is currently well-accepted and conducted in all neonates, especially those surviving neonatal intensive care. Nevertheless, this condition may be overlooked in critically ill children beyond the neonatal period even though they are exposed to similar hearing impairment risks. The early detection of hearing dysfunction might be beneficial to both the patients and the practitioners.

From this cohort, younger PICU survivors $(<12$ months of
Table 3. Multivariate analysis of associating factors for abnormal TEOAE responses

\begin{tabular}{lcc}
\hline Variable & Adjusted odds ratio (95\% Cl) & P-value \\
\hline Age $<12$ mo & $3.07(1.06-8.90)$ & 0.039 \\
Genetic abnormality & $6.95(1.49-32.54)$ & 0.014 \\
Aminoglycoside use & $1.82(0.23-14.15)$ & 0.566 \\
\hline
\end{tabular}

Adjusted for age $<12$ months, genetic abnormalities, and aminoglycoside use.

TEOAE: transitory evoked otoacoustic emission; $\mathrm{Cl}$ : confidence interval.

age) and those with genetic comorbidities were at higher risk for abnormal hearing screening responses. A higher negative impact of hearing dysfunction could be implicated in these children within the phase of speech development. Six children with underlying genetic conditions were found to have abnormal TEOAE responses. Children with genetic syndromes are often associated with hearing dysfunction, but these children had no previous history of abnormal hearing screening tests before PICU admission. It might also be possible that factors associated with critical illness might exacerbate underlying hearing dysfunction. Furthermore, children with genetic conditions might also suffer from other developmental problems, and hearing dysfunction might lead to further developmental dysfunction.

Unlike other previous studies, there was no association with abnormal hearing screening responses with diuretic use, ototoxic medication, positive pressure ventilation, epidural procedures, systemic infection or neurologic infection, prolonged mechanical ventilation, or traumatic brain injury in this study [1,3-12]. However, there was a non-statistically significant trend of abnormal TEOAE responses in children receiving aminoglycosides with a crude OR of 4.17 (95\% CI, 0.65-26.59). These non-statistically significant findings might be due to the small number of participants in this cohort receiving aminoglycosides. Due to the high crude OR for the trend of abnormal TEOAE responses with aminoglycoside utilization, aminoglycoside use was included in the multivariate analysis despite the non-statistically significant univariate analysis. The numbers of children receiving epidural procedures, having a neurologic infection, and traumatic brain injuries were also too small to obtain enough power to determine statistical significance.

There are several limitations to this study. The ABR should be conducted promptly at the same time as the abnormal TEOAE response to strengthen the confirmation of hearing dysfunction and to identify the locus of auditory pathology in 
the PICU survivors. Nonetheless, at our center, ABR was not readily available and thus required the long waiting period.

Furthermore, patient status and timing of TEOAE also played a role in the results of our study. There were still a number of PICU survivors who continued to require high flow nasal cannula support after being discharged from the PICU. High levels of ambient noise at the time of examination due to the high flow nasal cannula might have impeded testing, leading to various TEOAE screening timing intervals after PICU discharge. Despite our attempt to minimize the time of testing after PICU discharge, the median time for TEOAE testing was 7.0 days (IQR, 3.0-9.0 days). Thus, with varied timing, the intensive care management and environment might not fully explain the abnormal hearing screening responses. Nevertheless, we did not find a statistically significant difference in hospital admission duration among children with and without abnormal TEOAE responses. The proportion of children admitted to the hospital for more than 14 days were not statistically different among groups. Early testing might also underestimate or miss those with delayed-onset sensorineural hearing loss. A study by Fligor et al. [26] revealed that $48 \%$ of neonatal extracorporeal membrane graduates who received audiological screening had delayed-onset sensorineural hearing loss. Moreover, with a small population size for subgroup analysis, we might have underestimated the association of factors with an abnormal hearing screening.

Supratherapeutic serum concentrations and prolonged use of aminoglycosides for longer than 14 days were shown to increase patient risk of ototoxicity [26-28]. All patients within this cohort received aminoglycosides for more than seven days, but none received for greater than 14 days. Furthermore, serum aminoglycoside concentrations were not monitored, which led to another limitation of this study.

Despite limitations, our study demonstrates a high prevalence of abnormal hearing screening responses in children surviving critical illness, especially those younger than 12 months of age and those with genetic abnormalities. More extensive studies should be performed to further identify the associated risk factors of hearing problems in critically ill children. Further testing such as ABR is useful in confirming hearing loss and defines the nature and the severity of deficits. Additional study is needed to further elucidate the impact on communication, distress, and mental status of critically ill children with hearing loss.

\section{CONFLICT OF INTEREST}

No potential conflict of interest relevant to this article was reported.

\section{ACKNOWLEDGMENTS}

The authors show great appreciation to all the staff of our hospital for the strong support and extensive cooperation in making this study possible. The authors would also like to thank our clinical epidemiologist for statistical assistance. We also would like to express our gratitude to all parents and children participating in this study.

\section{ORCID}

Chanapai Chaiyakulsil https://orcid.org/0000-0003-4677-4223

\section{AUTHOR CONTRIBUTIONS}

Conceptualization: CC. Data curation: PS. Formal analysis: all authors. Methodology: all authors. Project administration: all authors. Visualization: CC. Writing-original draft: PS. Writingreview \& editing: all authors.

\section{REFERENCES}

1. Halpern NA, Pastores SM, Price JB, Alicea M. Hearing loss in critical care: an unappreciated phenomenon. Crit Care Med 1999;27:211-9.

2. Guyton A, Hall J. Sense of hearing. In: Hall J, editor. In: Textbook of medical physiology. Philadelphia (PA): W. B. Saunders; 1996. p. 663-73.

3. Fitzgerald DC. Head trauma: hearing loss and dizziness. J Trauma 1996;40:488-96.

4. Guo Y, Wu Y, Chen W, Lin J. Endotoxic damage to the stria vascularis: the pathogenesis of sensorineural hearing loss secondary to otitis media? J Laryngol Otol 1994;108:310-3.

5. Zeeshan F, Bari A, Dugal MN, Saeed F. Hearing impairment after acute bacterial meningitis in children. Pak J Med Sci 2018;34:655-9.

6. Davis LE, Johnsson LG. Viral infections of the inner ear: clinical, virologic, and pathologic studies in humans and animals. Am J Otolaryngol 1983;4:347-62.

7. Lerner SA, Matz GJ. Aminoglycoside ototoxicity. Am J Otolaryngol 1980;1:169-79. 
8. Masur H. Antimicrobials. In: Chernow B, editor. The pharmacologic approach to the critically ill patient. Baltimore (ML): Williams \& Wilkins; 1994. p. 685-99.

9. Brummett RE. Ototoxicity of vancomycin and analogues. Otolaryngol Clin North Am 1993;26:821-8.

10. Rybak LP. Ototoxicity of loop diuretics. Otolaryngol Clin North Am 1993;26:829-44.

11. Jung TT, Rhee CK, Lee CS, Park YS, Choi DC. Ototoxicity of salicylate, nonsteroidal antiinflammatory drugs, and quinine. Otolaryngol Clin North Am 1993;26:791-810.

12. Schaffartzik W, Hirsch J, Frickmann F, Kuhly P, Ernst A. Hearing loss after spinal and general anesthesia: a comparative study. Anesth Analg 2000;91:1466-72.

13. Neumann K, Indermark A. Validation of a new TEOAE-AABR device for newborn hearing screening. Int J Audiol 2012;51:5705.

14. Yin L, Bottrell C, Clarke N, Shacks J, Poulsen MK. Otoacoustic emissions: a valid, efficient first-line hearing screen for preschool children. J Sch Health 2009;79:147-52.

15. Johnson JL, White KR, Widen JE, Gravel JS, James M, Kennalley T, et al. A multicenter evaluation of how many infants with permanent hearing loss pass a two-stage otoacoustic emissions/ automated auditory brainstem response newborn hearing screening protocol. Pediatrics 2005;116:663-72.

16. van Dommelen P, Mohangoo AD, Verkerk PH, van der Ploeg CP, van Straaten HL; Dutch NICU Neonatal Hearing Screening Working Group. Risk indicators for hearing loss in infants treated in different neonatal intensive care units. Acta Paediatr 2010;99:344-9.

17. van Dommelen P, Verkerk PH, van Straaten HL; Dutch Neonatal Intensive Care Unit Neonatal Hearing Screening Working Group. Hearing loss by week of gestation and birth weight in very preterm neonates. J Pediatr 2015;166:840-3.

18. De Capua B, Costantini D, Martufi C, Latini G, Gentile M, De Felice C. Universal neonatal hearing screening: the Siena (Italy) experience on 19,700 newborns. Early Hum Dev 2007;83:601-6.

19.Eavey RD, Pinto LE, Thornton AR, Herrmann BS, do Carmo Bertero M, Saenz A. Early hearing testing of still critically ill neonates. Arch Otolaryngol Head Neck Surg 1996;122:289-93.

20. Cooper AC, Commers AR, Finkelstein M, Lipnik PG, Tollefson LM, Wilcox RA, et al. Otoacoustic emission screen results in critically ill neonates who received gentamicin in the first week of life. Pharmacotherapy 2011;31:649-57.

21. Hamill-Ruth RJ, Ruth RA, Googer K, Volles D, Deivert M, Turrentine B. Use of otoacoustic emissions to screen for hearing loss in critically ill patients. Audiology 1998;37:344-52.

22. Krug E, Cieza A, Chadha S, Sminkey L, Martinez R, Stevens G, et al. Childhood hearing loss: strategies for prevention and care. Geneva: World Health Organization; 2016.

23. Pollack MM, Patel KM, Ruttimann UE. PRISM III: an updated Pediatric Risk of Mortality score. Crit Care Med 1996;24:743-52.

24. Belletti A, Lerose CC, Zangrillo A, Landoni G. Vasoactive-Inotropic Score: evolution, clinical utility, and pitfalls. J Cardiothorac Vasc Anesth 2021;35:3067-77.

25. Quirk WS, Seidman MD. Cochlear vascular changes in response to loud noise. Am J Otol 1995;16:322-5.

26. Fligor BJ, Neault MW, Mullen CH, Feldman HA, Jones DT. Factors associated with sensorineural hearing loss among survivors of extracorporeal membrane oxygenation therapy. Pediatrics 2005;115:1519-28.

27. Black RE, Lau WK, Weinstein RJ, Young LS, Hewitt WL. Ototoxicity of amikacin. Antimicrob Agents Chemother 1976;9:956-61.

28. So TY. Use of ototoxic medications in neonates-the need for follow-up hearing test. J Pediatr Pharmacol Ther 2009;14:200-3. 